\title{
Quasi-Positive Delta Sequences and Their Applications in Wavelet Approximation
}

\author{
Shyam Lal and Susheel Kumar \\ Department of Mathematics, Institute of Science, Banaras Hindu University, Varanasi 221005, India \\ Correspondence should be addressed to Susheel Kumar; susphdbhu@gmail.com
}

Received 24 June 2016; Accepted 26 September 2016

Academic Editor: Birendra Nath Mandal

Copyright (C) 2016 S. Lal and S. Kumar. This is an open access article distributed under the Creative Commons Attribution License, which permits unrestricted use, distribution, and reproduction in any medium, provided the original work is properly cited.

\begin{abstract}
A sufficient literature is available for the wavelet error of approximation of certain functions in the $L^{2}$-norm. There is no work in context of multiresolution approximation of a function in the sense of sup-error. In this paper, for the first time, wavelet estimator for the approximation of a function $f$ belonging to $\operatorname{Lip}_{\alpha}[a, b]$ class under supremum norm has been obtained. Working in this direction, four new theorems on the wavelet approximation of a function $f$ belonging to $\operatorname{Lip}_{\alpha}, 0<\alpha \leq 1$ class using the projection $P_{m} f$ of its wavelet expansions have been estimated. The calculated estimator is best possible in wavelet analysis.
\end{abstract}

\section{Introduction}

Orthogonal wavelet is a new development in analysis but very useful in engineering and technology, especially in highresolution images and signal processing due to their localization properties in time and frequency. Wavelet expansions are superior to classical orthogonal series like Fourier series. Some properties of wavelet expansions have been studied by Chui [1], Daubechies and Lagarias [2], Meyer [3], Walter [4, 5], Islam et al. [6], and so forth. The idea of approximation of various functional spaces under different norms is obtained by Lal and Kumar [7, 8], Abu-Sirhan [9], Coskun [10], and Shiri and Azadi Kenary [11] which gives the inspiration for the present work. But till now no work seems to have been done to obtain the wavelet approximation of a function $f \in$ $\operatorname{Lip}_{\alpha}[a, b], 0<\alpha \leq 1$ using the projection $\left(P_{m} f\right)$ of its wavelet expansion and to discuss its convergence. In an attempt to make an advance study in this direction, in this paper, the best possible wavelet sup-error $E_{m} f$ of a function $f \in \operatorname{Lip}_{\alpha}[a, b]$ by $P_{m} f$ of its wavelet expansion has been determined. The convergence of wavelet expansions has been also discussed.

\section{Definitions and Preliminaries}

2.1. Multiresolution Analysis. Let $\mathbb{Z}$ be the set of all integers. A multiresolution analysis of $L^{2}(\mathbb{R})$ is defined as a sequence of closed subspaces $V_{j}$ of $L^{2}(\mathbb{R}), j \in \mathbb{Z}$, with the following properties:

(1) $V_{j} \subset V_{j+1}$.

(2) $f(x) \in V_{j} \Leftrightarrow f(2 x) \in V_{j+1}$.

(3) $f(x) \in V_{0} \Leftrightarrow f(x+1) \in V_{0}$.

(4) $\bigcup_{j=-\infty}^{\infty} V_{j}$ is dense in $L^{2}(\mathbb{R})$ and $\bigcap_{j=-\infty}^{\infty} V_{j}=\{0\}$.

(5) Suppose a function $\phi \in V_{0}$ exists such that the collection $\{\phi(x-k) ; k \in \mathbb{Z}\}$ is a Riesz basis of $V_{0}$.

Let $\psi \in L^{2}(\mathbb{R})$, and

$$
\begin{aligned}
& \psi_{j, k}:=2^{j / 2} \psi\left(2^{j} \cdot-k\right), \\
& W_{j}:=\operatorname{clos}\left\langle\psi_{j, k}: k \in \mathbb{Z}\right\rangle .
\end{aligned}
$$

Then this family of subspaces of $L^{2}(\mathbb{R})$ gives direct sum decomposition of $L^{2}(\mathbb{R})$ in the sense that every $f \in L^{2}(\mathbb{R})$ has unique decomposition:

$$
\begin{aligned}
f(x)= & \cdots+g_{-2}(x)+g_{-1}(x)+g_{0}(x)+g_{1}(x) \\
& +g_{2}(x)+\cdots,
\end{aligned}
$$

where $g_{j} \in W_{j}$ for all $j \in \mathbb{Z}$ and we describe this by writing

$$
L^{2}(\mathbb{R})=V_{j} \oplus_{i=j}^{\infty} W_{i},
$$


where

$$
V_{j}:=\oplus_{k=-\infty}^{j-1} W_{k}
$$

$\left\{\psi_{j, k} ; k \in \mathbb{Z}\right\}$ is a Riesz basis of $W_{j}$.

A function $\phi \in L^{2}(\mathbb{R})$ is called a scaling function, if the subspaces $V_{j}$ of $L^{2} \mathbb{R}$ defined by

$$
V_{j}:=\operatorname{clos}_{L^{2} \mathbb{R}}\left\{\phi_{j, k}: k \in \mathbb{Z}\right\}, \quad j \in \mathbb{Z}
$$

satisfy properties (1) to (5) stated above in this section. It is important to note that the scaling function $\phi$ generates a multiresolution analysis $\left\{V_{j}\right\}$ of $L^{2}(\mathbb{R})$ (Debnath [12]). tations

Following Walter [4], each $f \in L^{2}(\mathbb{R})$ has two represen-

$$
\begin{aligned}
f(t)= & \sum_{m=-\infty}^{\infty} \sum_{n=-\infty}^{\infty}\left\langle f, \psi_{m, n}\right\rangle \psi_{m, n}(t), \\
f(t)= & \sum_{n=-\infty}^{\infty}\left\langle f, \phi_{m, n}\right\rangle \phi_{m, n}(t) \\
& +\sum_{k=m}^{\infty} \sum_{n=-\infty}^{\infty}\left\langle f, \psi_{k, n}\right\rangle \psi_{k, n}(t) \\
= & \left(P_{m} f\right)(t)+\left(r_{m} f\right)(t),
\end{aligned}
$$

where convergence is in sense of $L^{2}(\mathbb{R})$. The function $\left(P_{m} f\right) \epsilon$ $V_{m}$ and in fact is the projection of $f$ onto $V_{m}$. It is also given in terms of the kernel $K_{m}(x, y)$ of $V_{m}$ as

$$
\left(P_{m} f\right)(x)=\int_{-\infty}^{\infty} K_{m}(x, t) f(t) d t .
$$

The function $K_{m}$ is given by

$$
K_{m}(x, y)=2^{m} K\left(2^{m} x, 2^{n} y\right),
$$

where

$$
\begin{aligned}
K(x, y) & =\sum_{n=-\infty}^{\infty} \phi(x-n) \bar{\phi}(y-n), \\
1 & =\int_{-\infty}^{\infty} \phi(x) d x=\sum_{n=-\infty}^{\infty} \phi(x-n) .
\end{aligned}
$$

It is remarkable to note that

$$
\begin{aligned}
K(x, y) & =\sum_{n=-\infty}^{\infty} \phi(x-n) \bar{\phi}(y-n) \\
K_{m}(x, y) & =2^{m} K\left(2^{m} x, 2^{m} y\right) \\
& =2^{m} \sum_{n=-\infty}^{\infty} \phi\left(2^{m} x-n\right) \bar{\phi}\left(2^{m} y-n\right) \\
& =\sum_{n=-\infty}^{\infty} 2^{m / 2} \phi\left(2^{m} x-n\right) 2^{m / 2} \bar{\phi}\left(2^{m} y-n\right) .
\end{aligned}
$$

Then

$$
\begin{aligned}
& \int_{-\infty}^{\infty} K_{m}(x, y) \\
& \quad=\sum_{n=-\infty}^{\infty} 2^{m}\left(\int_{-\infty}^{\infty} \phi\left(2^{m} x-n\right) d x\right) \bar{\phi}\left(2^{m} y-n\right)
\end{aligned}
$$

$$
\begin{aligned}
& =\sum_{n=-\infty}^{\infty}\left(\int_{-\infty}^{\infty} \phi(t) d t\right) \bar{\phi}\left(2^{m} y-n\right) \\
& =\sum_{n=-\infty}^{\infty} \bar{\phi}(y-n)=1 .
\end{aligned}
$$

\subsection{Quasi-Positive Delta Sequences. If}

(1) there is $C>0$ such that $\int_{-\infty}^{\infty}\left|\delta_{m}(x, y)\right| d x \leq C, y \in$ $\mathbb{R}, m \in \mathbb{N}$,

(2) $\int_{y-C}^{y+C} \delta_{m}(x, y) d x \rightarrow 1$ uniformly on compact subset of $\mathbb{R}$, as $m \rightarrow \infty$,

(3) for each $\gamma>0$,

$$
\sup _{|x-y| \geq \gamma}\left|\delta_{m}(x, y)\right| \longrightarrow 0 \quad \text { as } m \longrightarrow \infty
$$

then $\left\{\delta_{m}(\cdot, y)\right\}$ is known as quasi-positive delta sequence of function.

An example of a quasi-positive delta sequence is the Fejer kernel,

$$
\begin{aligned}
F_{m} & (x, y) \\
& =\frac{\sin ^{2}(((m+1) / 2)(x-y))}{2(m+1) \pi \sin ^{2}((x-y) / 2)} \chi_{[-\pi, \pi]}(x-y),
\end{aligned}
$$

while a delta sequence that is not quasi-positive is the Dirichlet kernel of Fourier series,

$$
D_{m}(x, y)=\frac{\sin (m+1 / 2)(x-y)}{2 \pi \sin ((x-y) / 2)} \chi_{[-\pi, \pi]}(x-y)
$$

because $\int_{-\infty}^{\infty} D_{m}(x, y) d x$ is not absolutely convergent.

2.3. Function of $\operatorname{Lip}_{\alpha}[a, b]$ Class. A function $f \in \operatorname{Lip}_{\alpha}[a, b]$ if

$$
|f(x)-f(y)|=O\left(|x-y|^{\alpha}\right), \quad \text { for } 0<\alpha \leq 1,
$$

(Titchmarsh [13], p. 406).

Examples

(1) If $f(x)=|x| \forall x \in[0,1], f \in \operatorname{Lip}_{\alpha}$.

(2) If $f(x)=1 / x \forall x \in(0,1], f \notin \operatorname{Lip}_{1}(0,1]$.

Let $N$ be a positive integer. Let $x=1 / N, y=1 /(N+$ 1) $x, y \in(0,1]$.

Then $x-y=1 / N-1 /(1+N)<1 / N$ and $1 /(x-y)>N$.

Now, $|f(x)-f(y)| /|x-y|=|N-(N+1)| /|x-y|=$ $1 /|x-y| \geq N$

$|f(x)-f(y)|>N|x-y|$

$\therefore f \notin \operatorname{Lip}_{1}(0,1]$. 
Remarks

(1) If $f \in \operatorname{Lip}_{\alpha}[a, b] \alpha>0$, then $f$ is continuous, indeed, uniformly continuous on $[a, b]$.

(2) $\operatorname{Lip}_{\alpha}$ class is a linear space over $\mathbb{R}$ or $\mathbb{C}$.

(3) If $f \in \operatorname{Lip}_{\alpha} \alpha>1$, then $f$ is constant function.

2.4. Projection $P_{m} f . P_{m} f$, the orthogonal projection of $L^{2}(\mathbb{R})$ onto $V_{m}$, is defined by

$$
P_{m} f=\sum_{k=-\infty}^{\infty}\left\langle f, \phi_{m, k}\right\rangle \phi_{m, k}, \quad m \in \mathbb{Z},
$$

(Sweldens and Piessens [14]).

2.5. Wavelet Approximation. The wavelet approximation $E_{m}(f)$ of a function $f$ under supremum norm is defined by

$$
E_{m}(f)=\left\|f-P_{m} f\right\|_{\infty}=\sup _{x}\left|\left(f(x)-P_{m} f(x)\right)\right|
$$

(Zygmund [15], p. 114).

If $E_{m}(f) \rightarrow 0$ as $m \rightarrow \infty$ then $E_{m}(f)$ is called the best approximation of $f$ of order $m$ (Zygmund [15], p. 115).

\section{Theorems}

In this paper, we prove the following theorems.

Theorem 11. Let $\phi \in L^{2}(\mathbb{R})$ be a scaling function and $\psi \epsilon$ $L^{2}(\mathbb{R})$ be a basic wavelet satisfying the admissibility condition

$$
C_{\psi}=\int_{-\infty}^{\infty} \frac{|\widehat{\psi}(\omega)|^{2}}{|\omega|} d \omega<\infty .
$$

If a function $f \in \operatorname{Lip}_{\alpha}[a, b]$ is represented by its wavelet expansion as

$$
\begin{aligned}
f(\cdot)= & \sum_{n=-\infty}^{\infty}\left\langle f, \phi_{m, n}\right\rangle \phi_{m, n}(\cdot) \\
& +\sum_{k=m}^{\infty} \sum_{n=-\infty}^{\infty}\left\langle f, \psi_{m, n}\right\rangle \psi_{m, n}(\cdot) \\
= & \left(P_{m} f\right)(\cdot)+\left(r_{m} f\right)(\cdot)
\end{aligned}
$$

then the wavelet approximation $E_{m}(f)$ of $f$ by $\left(P_{m} f\right)$ satisfies

$$
\begin{aligned}
E_{m}(f) & =\left\|f-P_{m} f\right\|_{\infty}=\sup _{y \in[a, b]}\left|(f(y))-P_{m} f(y)\right| \\
& =O\left(\frac{1}{(|m|+1)^{\alpha}}\right) .
\end{aligned}
$$

Theorem 12. If a function $f \in \operatorname{Lip}_{\alpha}[a, b]$ and $P_{m} f$ is the projection of $f$ onto $V_{m}$ then $P_{m} f \rightarrow f$ as $m \rightarrow \infty$ uniformly on $[a, b]$.
Theorem 13. If $f \in \operatorname{Lip}_{\alpha}[a, b]$ and

$$
f(\cdot)=\left(P_{m} f\right)(\cdot)+\left(r_{m} f\right)(\cdot)
$$

then

$$
\left\|r_{m} f\right\|_{\infty}=O\left(\frac{1}{(|m|+1)^{\alpha}}\right), \quad 0<\alpha \leq 1 .
$$

Theorem 14. If $f \in \operatorname{Lip}_{\alpha}[a, b]$,

$$
f(\cdot)=\left(P_{m} f\right)(\cdot)+\left(r_{m} f\right)(\cdot),
$$

then $r_{m} f \rightarrow 0$ as $m \rightarrow \infty$ uniformly on $[a, b]$.

\section{Lemma}

For the proof of our theorems, the following lemma is required.

Lemma 15. If $K_{m}(x, y)=2^{m} K\left(2^{m} x, 2^{m} y\right), x, y \in[a, b]$, then $\left\{K_{m}(\cdot, y)\right\}_{-\infty}^{\infty}$ is a quasi-positive delta sequence of function in $L^{1}(\mathbb{R})$.

4.1. Proof of Lemma 15. Following the proof of Walter ([4] p. 113),

$$
\int_{-\infty}^{\infty}\left|K_{m}(x, y)\right| d x=C
$$

Let $c>0$; then

$$
\begin{aligned}
\int_{y-c}^{y+c}\left|K_{m}(x, y)\right| d x= & -\int_{-\infty}^{y-2^{m} c}|K(t, y)| d t \\
& -\int_{y+2^{m} c}^{\infty}|K(t, y)| d t
\end{aligned}
$$

$\int_{-\infty}^{y-2^{m} c}|K(t, y)| d t \rightarrow 0$ as $m \rightarrow \infty$ and $\int_{y+2^{m} c}^{\infty}|K(t, y)| d t \rightarrow 0$ as $m \rightarrow \infty$.

Thus, $\int_{y-c}^{y+c}\left|K_{m}(x, y)\right| d x \rightarrow 1$ as $m \rightarrow \infty$ uniformly on $[a, b] \subset \mathbb{R}$ and $\sup _{|x-y| \geq 1 /(|m|+1)}\left|K_{m}(x, y)\right| \rightarrow 0$ as $m \rightarrow \infty$, [4].

Therefore $\left\{K_{m}(x, y)\right\}_{-\infty}^{\infty}$ is a quasi-positive delta sequence of functions.

\section{Proofs}

5.1. Proof of Theorem 11. $\left(P_{m} f\right)$ is given by

$$
\begin{aligned}
& \left(P_{m} f\right)(y)=\sum_{n=-\infty}^{\infty}\left\langle f, \phi_{m, n}\right\rangle \phi_{m, n}(y) \\
& =\sum_{n=-\infty}^{\infty}\left(\int_{-\infty}^{\infty} f(x) \bar{\phi}_{m, n}(x) d x\right) \phi_{m, n}(y) \\
& =\int_{-\infty}^{\infty} f(x)\left(\sum_{n=-\infty}^{\infty} \phi_{m, n}(y) \overline{\phi_{m, n}(x)}\right) d x \\
& =\int_{-\infty}^{\infty} f(x) \\
& \cdot\left(\sum_{n=-\infty}^{\infty} 2^{m / 2} \phi\left(2^{m} y-n\right) 2^{m / 2} \frac{\phi\left(2^{m} x-n\right)}{\phi}\right) d x
\end{aligned}
$$




$$
\begin{aligned}
& =\int_{-\infty}^{\infty} f(x) \\
& \left(\sum_{n=-\infty}^{\infty} 2^{m} \phi\left(2^{m} y-n\right) \overline{\phi\left(2^{m} x-n\right)}\right) d x \\
& =\int_{-\infty}^{\infty} f(x) 2^{m} K\left(2^{m} x, 2^{m} y\right) d x \\
& =\int_{-\infty}^{\infty} K_{m}(x, y) f(x) d x \\
& f(y)-\left(P_{m} f\right)(y)=f(y)-\int_{-\infty}^{\infty} K_{m}(x, y) f(x) d x \\
& =f(y) \int_{-\infty}^{\infty} K_{m}(x, y) d x-\int_{-\infty}^{\infty} K_{m}(x, y) \\
& f(x) d x, \quad \int_{-\infty}^{\infty} K_{m}(x, y) d x=1 \\
& =\int_{-\infty}^{\infty} K_{m}(x, y)(f(y)-f(x)) d x \\
& =\int_{-\infty}^{y-1 /(|m|+1)} K_{m}(x, y)(f(y)-f(x)) \\
& +\int_{y-1 /(|m|+1)}^{y+1 /(|m|+1)} K_{m}(x, y)(f(y)-f(x)) d x \\
& +\int_{y+1 /(|m|+1)}^{\infty} K_{m}(x, y)(f(y)-f(x)) \\
& \left|f(y)-\left(P_{m} f\right)(y)\right| \leq \int_{-\infty}^{y-1 /(|m|+1)}\left|K_{m}(x, y)\right| \\
& \cdot|f(y)-f(x)|+\int_{y-1 /(|m|+1)}^{y+1 /(|m|+1)}\left|K_{m}(x, y)\right| \\
& \cdot|(f(y)-f(x))| d x+\int_{y+1 /(|m|+1)}^{\infty}\left|K_{m}(x, y)\right| \\
& \cdot|(f(y)-f(x))| d x=I_{1}+I_{2}+I_{3} \text { say. }
\end{aligned}
$$

Let $x, y \in[a, b]$. Select $m$ such that

$$
\begin{aligned}
& y+\frac{1}{|m|+1}<b, \\
& x-\frac{1}{|m|+1}>a .
\end{aligned}
$$

Since it is known that

$$
\begin{gathered}
\sup _{x \in[y-1 /(|m|+1), y+1 /(|m|+1)]}|f(x)-f(y)| \\
\leq\left|f\left(y+\frac{1}{|m|+1}\right)-f(y)\right| \\
+\left|f\left(y-\frac{1}{|m|+1}\right)-f(y)\right|
\end{gathered}
$$

$$
\begin{aligned}
& =M_{1}\left(\frac{1}{|m|+1}\right)^{\alpha}+M_{2}\left(\frac{1}{|m|+1}\right)^{\alpha}, \quad f \in \operatorname{Lip}_{\alpha} \\
& =\left(M_{1}+M_{2}\right) \frac{1}{(|m|+1)^{\alpha}} \quad \text { where } M_{1}>0, M_{2}>0
\end{aligned}
$$

therefore

$$
\begin{aligned}
& I_{2}=\int_{y-1 /(|m|+1)}^{y+1 /(|m|+1)}\left|K_{m}(x, y)\right||(f(x)-f(y))| d x \\
& \leq \frac{M_{1}+M_{2}}{(|m|+1)^{\alpha}} \int_{y-1 /(|m|+1)}^{y+1 /(|m|+1)}\left|K_{m}(x, y)\right| d x \\
& \leq \frac{M_{1}+M_{2}}{(|m|+1)^{\alpha}} \int_{-\infty}^{\infty}\left|K_{m}(x, y)\right| d x=\frac{\left(M_{1}+M_{2}\right) C}{(|m|+1)^{\alpha}}, \\
& C>0 \text { by Lemma } 15 .
\end{aligned}
$$

Next,

$$
\begin{aligned}
I_{1} & +I_{3}=\int_{-\infty}^{y-1 /(|m|+1)}\left|K_{m}(x, y)\right||f(y)-f(x)| d x \\
& +\int_{y+1 /(|m|+1)}^{\infty}\left|K_{m}(x, y)\right||f(y)-f(x)| d x \\
& =\int_{a}^{y-1 /(|m|+1)}\left|K_{m}(x, y)\right||f(y)-f(x)| d x \\
& +\int_{y+1 /(|m|+1)}^{b}\left|K_{m}(x, y)\right||f(y)-f(x)| d x \\
& \leq \sup _{|x-y| \geq 1 /(|m|+1)}\left|K_{m}(x, y)\right| \\
& \cdot \frac{M_{3}}{(|m|+1)^{\alpha}}\left(\int_{a}^{y-1 /(|m|+1)} d x+\int_{y+1 /(|m|+1)}^{b} d x\right), \\
\leq & \frac{M_{3}(b-a) C_{1}}{(|m|+1)^{\alpha}, C_{1}>0 .} M_{3}>0 \\
& \frac{\sup _{3}}{(|m|+1 /(|m|+1)}\left|K_{m}(x, y)\right| \\
& \left\{(b-a)-\frac{2}{|m|+1}\right\},
\end{aligned}
$$

$\left\{K_{m}(x, y)\right\}$ is a quasi-positive delta sequence of function. Now, collecting (50), (31), and (32), we have

$$
\begin{aligned}
\left|f(y)-\left(P_{m} f\right)(y)\right| \leq & \frac{M_{3}(b-a) C_{1}}{(|m|+1)^{\alpha}} \\
& +\frac{\left(M_{1}+M_{2}\right) C}{(|m|+1)^{\alpha}}
\end{aligned}
$$




$$
\begin{aligned}
& =\frac{M_{3}(b-a) C_{1}+\left(M_{1}+M_{2}\right) C}{(|m|+1)^{\alpha}} \\
& =O\left(\frac{1}{(|m|+1)^{\alpha}}\right) .
\end{aligned}
$$

Thus

$$
\begin{aligned}
\left\|f-P_{m} f\right\|_{\infty} & =\sup _{y \in[a, b]}\left|f(y)-P_{m} f(y)\right| \\
& =O\left(\frac{1}{(|m|+1)^{\alpha}}\right) .
\end{aligned}
$$

Remark 16 (the converse of Theorem 11 is also true). Choose $x, y \in[a, b]$ such that $x-1 /(|m|+1), y+1 /(|m|+1) \in[a, b]$ and $|y-x|=d /(|m|+1), d>0$.

$$
\begin{aligned}
& |f(x)-f(y)|=\mid f(x)-\left(P_{m} f\right)(x)+\left(P_{m} f\right)(x) \\
& \quad-\left(P_{m} f\right)(y)+\left(P_{m} f\right)(y)-f(y)|\leq| f(x) \\
& \quad-\left(P_{m} f\right)(x)|+|\left(P_{m} f\right)(x)-\left(P_{m} f\right)(y) \mid \\
& \quad+\left|\left(P_{m} f\right)(y)-f(y)\right| \leq \sup _{x \in[a, b]}\left|f(x)-\left(P_{m} f\right)(x)\right| \\
& \quad+\sup _{x, y \in[a, b]}\left|\left(P_{m} f\right)(x)-\left(P_{m} f\right)(y)\right| \\
& \quad+\sup _{y \in[a, b]}\left|\left(P_{m} f\right)(y)-f(y)\right|=\left\|f-P_{m} f\right\|_{\infty} \\
& +\sup _{x, y \in[a, b]}\left|\left(P_{m} f\right)(x-y)\right|+\left\|P_{m} f-f\right\|_{\infty} \\
& \quad=O\left(\frac{1}{(|m|+1)^{\alpha}}\right)+O\left(|x-y|^{\alpha}\right) \\
& \quad+O\left(\frac{1}{(|m|+1)^{\alpha}}\right)=O\left(\frac{1}{(|m|+1)^{\alpha}}\right) \\
& \quad+O\left(\frac{1}{(|m|+1)^{\alpha}}\right)+O\left(\frac{1}{(|m|+1)^{\alpha}}\right) \\
& \quad=O\left(|x-y|^{\alpha}\right) .
\end{aligned}
$$

Since $|f(x)-f(y)|=O|x-y|^{\alpha}$, therefore $f \in \operatorname{Lip}_{\alpha}[a, b]$.

Hence, Theorem 11 is completely established.

5.2. Proof of Theorem 12. By the proof of Theorem 11

$$
\left\|P_{m} f-f\right\|_{\infty}=O\left(\frac{1}{(|m|+1)^{\alpha}}\right) .
$$

Since,

$$
\frac{1}{(|m|+1)^{\alpha}} \longrightarrow 0 \quad \text { as } m \longrightarrow \infty
$$

therefore,

$$
\left\|P_{m} f-f\right\|_{\infty} \longrightarrow 0 \text { as } m \longrightarrow \infty .
$$

Thus,

$$
P_{m} f \longrightarrow f \quad \text { as } m \longrightarrow \infty \text { uniformly on }[a, b] .
$$

Hence, Theorem 12 is completely established.

5.3. Proof of Theorem 13. Since,

$$
f(x)=P_{m} f(x)+r_{m} f(x)
$$

therefore,

$$
\begin{aligned}
r_{m} f(x) & =f(x)-P_{m} f(x), \\
\left\|r_{m} f\right\|_{\infty} & =\left\|f-P_{m} f\right\|_{\infty} \\
& =O\left(\frac{1}{(|m|+1)^{\alpha}}\right), \text { by Theorem } 11 .
\end{aligned}
$$

Hence, Theorem 13 is completely established.

5.4. Proof of Theorem 14. Following the proof of Theorem 13,

$$
\left\|r_{m} f\right\|_{\infty}=O\left(\frac{1}{(|m|+1)^{\alpha}}\right) .
$$

Since,

$$
\left\|r_{m} f\right\|_{\infty} \longrightarrow 0 \text { as } m \longrightarrow \infty
$$

therefore,

$$
r_{m} f \longrightarrow 0 \quad \text { as } m \longrightarrow \infty \text { uniformly on }[a, b] .
$$

Hence, Theorem 14 is completely established.

\section{Notes}

(1) $E_{m}(f) \rightarrow 0$ as $m \rightarrow \infty$ in Theorem 11; the wavelet approximations determined in this theorem are best possible in wavelet analysis (Zygmund, [15], p. 115).

(2) Define a function $\phi:[0,1] \rightarrow \mathbb{R}$ by

$$
\begin{aligned}
\phi(t) & =|t-1|^{\alpha}-\left|t^{\alpha}-1\right| \quad \forall t \in[0,1], 0<\alpha<1 \\
& =(1-t)^{\alpha}-\left(1-t^{\alpha}\right) \\
& =(1-t)^{\alpha}+t^{\alpha}-1 \quad \forall t \in[0,1] . \\
\therefore \phi^{\prime}(t) & =-\alpha(1-t)^{\alpha-1}+\alpha t^{\alpha-1} \quad \forall t \in(0,1) \\
& =\alpha\left(\frac{1}{t^{1-\alpha}}-\frac{1}{(1-t)^{1-\alpha}}\right) \\
\therefore \phi^{\prime}(t) & >0, \quad \forall t \in\left(0, \frac{1}{2}\right) \\
& =0, \quad t=\frac{1}{2} \\
& <0, \quad \forall t \in\left(\frac{1}{2}, 1\right) .
\end{aligned}
$$


Therefore $\phi$ is monotonic increasing in $(0,1 / 2)$ and monotonic decreasing in $(1 / 2,1)$

$$
\begin{gathered}
\therefore \phi(t) \geq \phi(0)=\phi(1)=0 \quad \forall t \in(0,1) \\
\therefore|t-1|^{\alpha}-\left|t^{\alpha}-1\right| \geq 0 \\
\therefore|t-1|^{\alpha} \geq\left|t^{\alpha}-1\right| .
\end{gathered}
$$

Thus,

$$
\begin{gathered}
\left|\frac{x}{y}-1\right|^{\alpha} \geq\left|\left(\frac{x}{y}\right)^{\alpha}-1\right| \\
t=\frac{x}{y}
\end{gathered}
$$

$$
0<x, y<1
$$

Hence $|x-y|^{\alpha} \geq\left|x^{\alpha}-y^{\alpha}\right|$

$$
\text { that is }\left|x^{\alpha}-y^{\alpha}\right| \leq|x-y|^{\alpha} \quad 0<x, y<1 \text {. }
$$

A function $f:[a, b] \rightarrow \mathbb{R}$ is defined by $f(x)=\left(1-x^{2}\right)^{\alpha}$, $0<\alpha<1, \forall x \in[a, b] \subset[0,1]$.

$$
\begin{aligned}
|f(x)-f(y)| & =\left|\left(1-x^{2}\right)^{\alpha}-\left(1-y^{2}\right)^{\alpha}\right| \\
& \leq\left|\left(1-x^{2}\right)-\left(1-y^{2}\right)\right|^{\alpha} \\
& =\left|y^{2}-x^{2}\right|^{\alpha}=|x-y|^{\alpha}|x+y|^{\alpha} \\
& \leq|x-y|^{\alpha}\left\{|x|^{\alpha}+|y|^{\alpha}\right\} \\
& \leq \max \left\{|2 a|^{\alpha},|2 b|^{\alpha}\right\}|x-y|^{\alpha} \\
& \leq 2^{\alpha}\left(|a|^{\alpha}+|b|^{\alpha}\right)|x-y|^{\alpha} \\
& =O\left(|x-y|^{\alpha}\right)
\end{aligned}
$$

$\therefore f \in \operatorname{Lip}_{\alpha}[a, b], 0<\alpha<1$.

For this function,

$$
\begin{aligned}
& \left|f(y)-\left(P_{m} f\right)(y)\right| \\
& \quad \leq \int_{-\infty}^{y-1 /(|m|+1)}\left|K_{m}(x, y)\right||f(y)-f(x)| d x \\
& \quad+\int_{y-1 /(|m|+1)}^{y+1 /(|m|+1)}\left|K_{m}(x, y)\right||(f(y)-f(x))| d x \\
& \quad+\int_{y+1 /(|m|+1)}^{\infty}\left|K_{m}(x, y)\right||(f(y)-f(x))| d x \\
& =I_{1}+I_{2}+I_{3} \text { say. }
\end{aligned}
$$

Then

$$
\begin{aligned}
& I_{2}=\int_{y-1 /(|m|+1)}^{y+1 /(|m|+1)}\left|K_{m}(x, y)\right||x-y|^{\alpha}|x+y|^{\alpha} d x \\
& \leq 2^{\alpha}\left(|a|^{\alpha}+|b|^{\alpha}\right) \int_{y-1 /(|m|+1)}^{y+1 /(|m|+1)}\left|K_{m}(x, y)\right||x-y|^{\alpha} d x,
\end{aligned}
$$

$$
|x+y|^{\alpha} \leq 2^{\alpha}\left(|a|^{\alpha}+|b|^{\alpha}\right)
$$

$$
\begin{array}{r}
\leq \frac{2^{2 \alpha}\left(|a|^{\alpha}+|b|^{\alpha}\right)}{(|m|+1)^{\alpha}} \int_{y-1 /(|m|+1)}^{y+1 /(|m|+1)}\left|K_{m}(x, y)\right| d x, \\
|x-y| \leq \frac{2}{|m|+1}
\end{array}
$$

$$
\begin{aligned}
& \leq \frac{2^{2 \alpha}\left(|a|^{\alpha}+|b|^{\alpha}\right)}{(|m|+1)^{\alpha}} \int_{-\infty}^{\infty}\left|K_{m}(x, y)\right| d x \\
& =\frac{2^{2 \alpha}\left(|a|^{\alpha}+|b|^{\alpha}\right)}{(|m|+1)^{\alpha}}, \quad \int_{-\infty}^{\infty}\left|K_{m}(x, y)\right| d x=1 \\
& =O\left(\frac{1}{(|m|+1)^{\alpha}}\right)
\end{aligned}
$$

$$
\begin{aligned}
I_{1} & +I_{3}=\int_{a}^{y-1 /(|m|+1)}\left|K_{m}(x, y)\right||f(y)-f(x)| d x \\
& +\int_{y+1 /(|m|+1)}^{b}\left|K_{m}(x, y)\right||f(y)-f(x)| d x \\
& \leq \sup _{|x-y| \geq 1 /(|m|+1)}\left|K_{m}(x, y)\right| \frac{2^{\alpha}\left(|a|^{\alpha}+|b|^{\alpha}\right)}{(|m|+1)^{\alpha}} \\
& \cdot\left(\int_{a}^{y-1 /(|m|+1)} d x+\int_{y+1 /(|m|+1)}^{b} d x\right) \\
& \leq \sup _{|x-y| \geq 1 /(|m|+1)}\left|K_{m}(x, y)\right| \frac{2^{\alpha}\left(|a|^{\alpha}+|b|^{\alpha}\right)}{(|m|+1)^{\alpha}}(b-a) \\
& =O\left(\frac{1}{(|m|+1)^{\alpha}}\right) .
\end{aligned}
$$

Hence

$$
\sup _{y \in[a, b]}\left|f(y)-P_{m} f(y)\right|=O\left(\frac{1}{(|m|+1)^{\alpha}}\right) .
$$

$\left\|f(y)-P_{m} f(y)\right\|_{\infty}=O\left(1 /(|m|+1)^{\alpha}\right)$.

Thus, the result of Theorem 11 is verified for a function $f$ defined by $f(x)=\left(1-x^{2}\right)^{\alpha}, 0<\alpha<1$.

(3) Hölder class: Let $C_{2 \pi}$ denote the Banach spaces of all $2 \pi$ periodic continuous functions under sup-norm. For each $0<\alpha \leq 1$ and some positive constant $k$, the function space $H_{\alpha}$ is defined by

$$
H_{\alpha}=\left\{f \in C_{2 \pi}:|f(x)-f(y)| \leq k|x-y|^{\alpha}\right\}
$$

(Das et al. [16] p. 83).

Let $f \in H_{\alpha}$. Then

$$
|f(x)-f(y)|=k|x-y|^{\alpha}=O\left(|x-y|^{\alpha}\right)
$$

$\therefore f \in \operatorname{Lip}_{\alpha}$. 
The function $f(x)=\left(1-x^{3}\right)^{\alpha}, 0<\alpha<1, \forall x \in[0,1]$. It is continuous and belonging to $\operatorname{Lip}_{\alpha}$ but $f(x+2 \pi) \neq f(x)$. Then $f \notin H_{\alpha}$.

Thus $H_{\alpha} \varsubsetneqq \operatorname{Lip}_{\alpha}$.

Theorem 11 is also valid for a function $f \in H_{\alpha}$.

\section{Competing Interests}

The authors declare that they have no competing interests.

\section{Acknowledgments}

Shyam Lal is thankful to DST-CIMS for encouragement to this work. Susheel Kumar is grateful to CSIR (India) in the form of Junior Research Fellowship with Ref. no. 19-06/2011 (i)EU-IV dated 03-10-2011 for this research work.

\section{References}

[1] C. K. Chui, An Introduction to Wavelets, vol. 1 of Wavelet Analysis and Its Applications, Academic Press, Boston, Mass, USA, 1992.

[2] I. Daubechies and J. C. Lagarias, "Two-scale difference equations. I. Existence and global regularity of solutions," SIAM Journal on Mathematical Analysis, vol. 22, no. 5, pp. 1388-1410, 1991.

[3] Y. Meyer, "Wavelets their post and their future," in Progress in Wavelet Analysis and Applications (Toulouse, 1992), Y. Meyer and S. Roques, Eds., pp. 9-18, Frontieres, Gif-Sur-Yvette, France, 1993.

[4] G. G. Walter, "Pointwise convergence of wavelet expansions," Journal of Approximation Theory, vol. 80, no. 1, pp. 108-118, 1995.

[5] G. G. Walter, "Approximation of the delta function by wavelets," Journal of Approximation Theory, vol. 71, no. 3, pp. 329-343, 1992.

[6] M. R. Islam, S. F. Ahemmed, and S. M. Rahman, "Comparison of wavelet approximation order in different smoothness spaces," International Journal of Mathematics and Mathematical Sciences, vol. 2006, Article ID 63670, 7 pages, 2006.

[7] S. Lal and M. Kumar, "Approximation of functions of space $L^{2}(\mathbb{R})$ by wavelet expansions," Lobachevskii Journal of Mathematics, vol. 34, no. 2, pp. 163-172, 2013.

[8] S. Lal and S. Kumar, "Best wavelet approximation of functions belonging to generalized lipschitz class using haar scaling function," Thai Journal of Mathematics, In press.

[9] E. Abu-Sirhan, "Best simultaneous approximation in function and operator spaces," Turkish Journal of Mathematics, vol. 36, no. 1, pp. 101-112, 2012.

[10] T. Coskun, "On the order of weighted approximation by positive linear operators," Turkish Journal of Mathematics, vol. 36, no. 1, pp. 113-120, 2012.

[11] M. S. Shiri and H. Azadi Kenary, "Approximation of an additive mapping in various normed spaces," Iranian Mathematical Society, vol. 41, no. 5, pp. 1213-1233, 2015.

[12] L. Debnath, Wavelet Transforms and Their Applications, Birkhäuser, Boston, Mass, USA, 2002.

[13] E. C. Titchmarsh, The Theory of Functions, Oxford University Press, Oxford, UK, 2nd edition, 1939.

[14] W. Sweldens and R. Piessens, "Quadrature formulae and asymptotic error expansions for wavelet approximations of smooth functions," SIAM Journal on Numerical Analysis, vol. 31, no. 4, pp. 1240-1264, 1994.

[15] A. Zygmund, Trigonometric Series Volume I, Cambridge University Press, 1959.

[16] D. Das, S. Mishra, and B. K. Ray, "Degree of approximation by Euler means of $\mathrm{r}$-th derived Fourier in the holder metric," Journal of National Academy of Mathematics India, vol. 10, 19921996. 


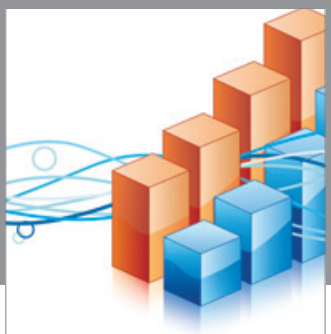

Advances in

Operations Research

vatem alat4

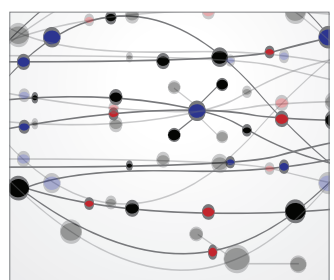

\section{The Scientific} World Journal
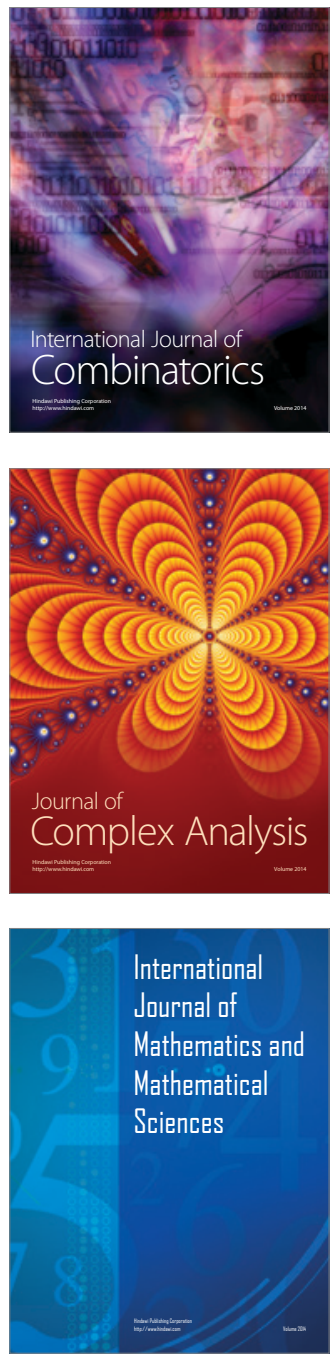
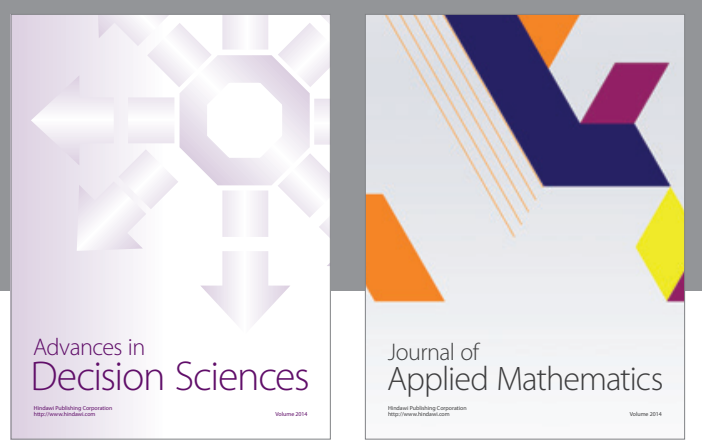

Algebra

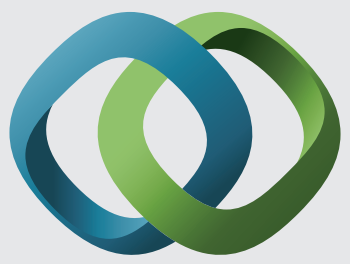

\section{Hindawi}

Submit your manuscripts at

http://www.hindawi.com
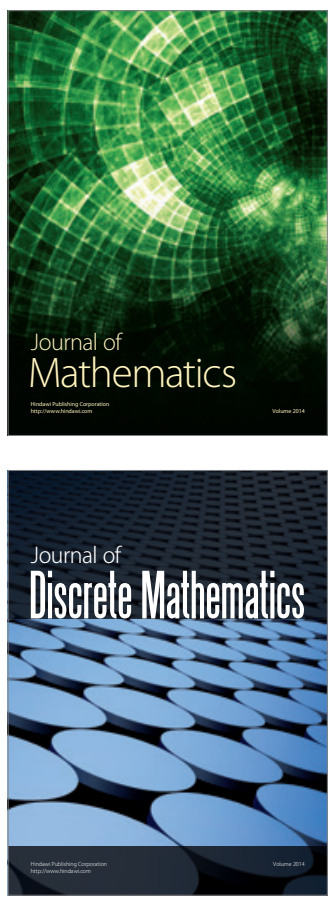

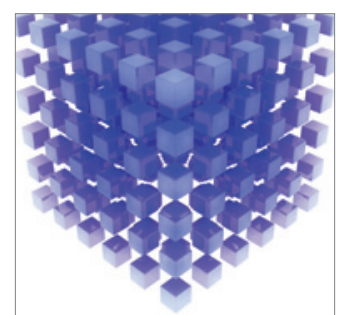

Mathematical Problems in Engineering
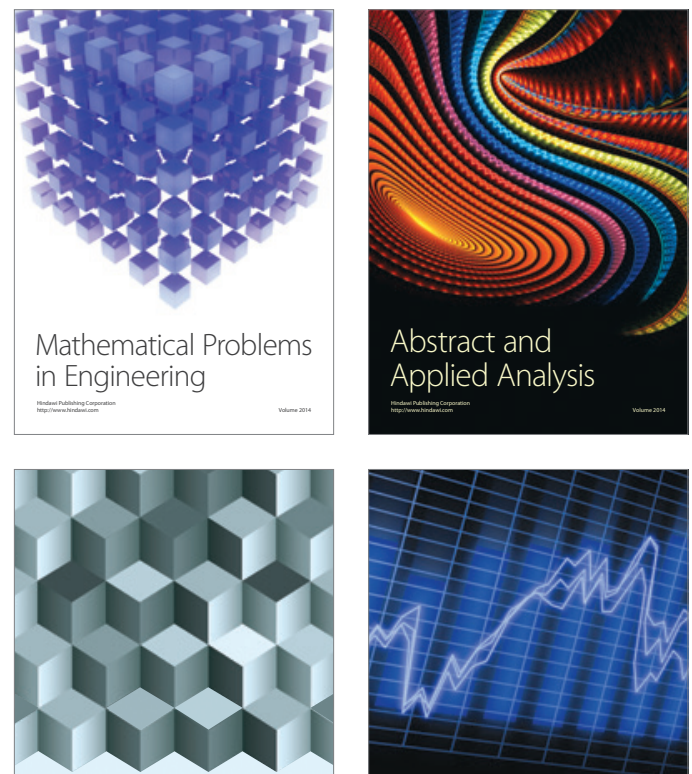

Journal of

Function Spaces

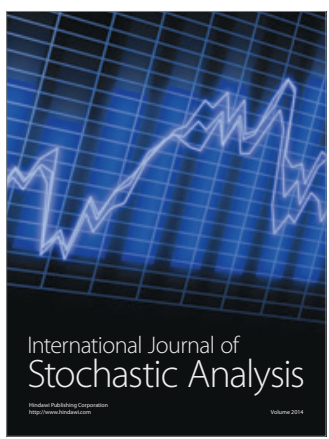

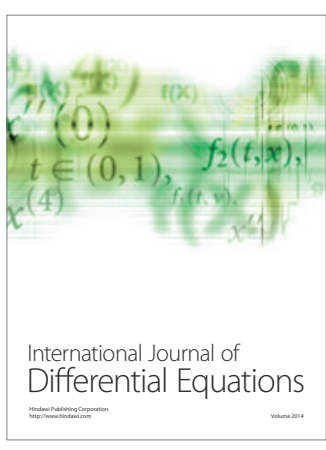
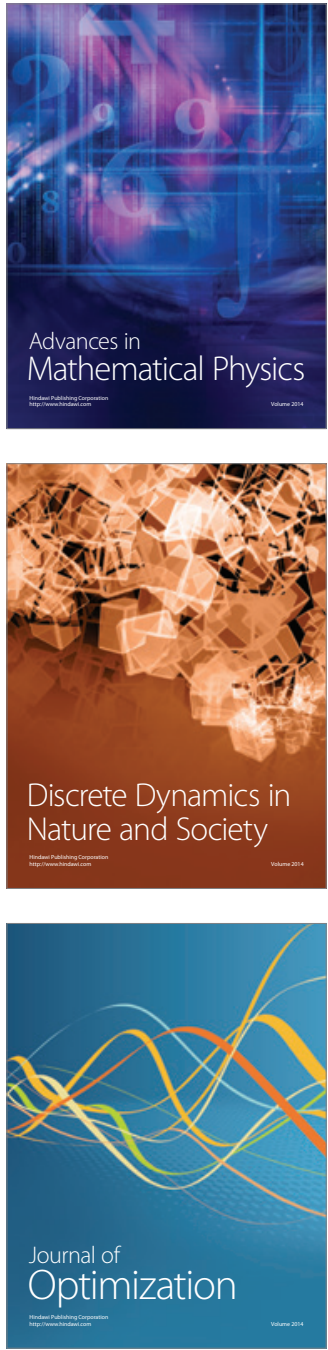